\title{
Physiologic Studies of Dysmetria in Patients with Cerebellar Deficits
}

\author{
Mark Hallett and Steve G. Massaquoi
}

\begin{abstract}
A feature of cerebellar ataxia is dysmetria, which is characterized by inaccurate movements. Studies of rapid movements at a single joint show prolonged acceleration phases and prolonged initial bursts of EMG activity in the agonist muscle. These two features correlate with each other, suggesting that the prolongation of the neural signal is responsible for the kinematic abnormality. This explains a tendency to hypermetria. Studies of multijoint movements show abnormalities in relative timing of the different joints. During locomotion, knee and ankle motions can be delayed differentially with respect to the gait cycle. Subjects attempting straight-line movements with the arm have systematic deviations that reflect incoordination of the shoulder and elbow with respect to each other. A possible explanation of dysmetria is a failure of sufficient force generation within the necessary time to accomplish a coordinated movement. Another possible explanation is that the cerebellum is responsible for timing of brain functions.
\end{abstract}

RÉSUMÉ: Études physiologiques de la dysmétrie chez des patients avec déficits cérébelleux. La dysmétrie est une manifestation de l'ataxie cérébelleuse qui se caractérise par des mouvements imprécis. Des études de mouvements rapides impliquant une seule articulation ont montré des phases d'accélération prolongées ainsi que des salves initiales prolongées d'activité EMG dans les muscles agonistes. Ces deux particularités sont correlées entre elles, suggérant que la prolongation du signal nerveux est responsable de l'anomalie cinématique expliquant la tendance à l'hypermétrie. L'étude de mouvements impliquant plusieurs articulations montre des anomalies dans la synchronisation relative des différentes articulations. A la marche, les mouvements du genou et de la cheville peuvent être retardés différemment par rapport au cycle de la marche. A l'exécution de mouvements en ligne droite avec le bras, on observe des déviations systématiques qui reflètent l'incoordination de l'épaule et du coude par rapport l'une à l'autre. L'incapacité de générer une force suffisante dans le temps voulu pour accomplir un mouvement coordonné est une explication possible de la dysmétrie, une autre explication étant que le cervelet est responsable de la synchronisation des fonctions cérébrales.

Can.J. Neurol. Sci. 1993; 20 (Suppl. 3): S83-S92

It is commonly accepted that the cerebellum plays a role in the coordination of movement. The details of its contribution to the control of movement, however, remain vague. In spite of the well-known problems associated with studying the inference of brain function by the analysis of the effects of lesions, the method continues to be of value. Additionally, it is useful in clinical practice to understand the pathophysiology of neurologic disorders. Patients with lesions of the cerebellum are said to be "ataxic," but this term simply means incoordination of the cerebellar type. The patients manifest many different signs and symptoms, and while these may reflect multiple disorders of the cerebellum, it may well also be the case that there are only a few fundamental disorders (or possibly even one) that are responsible for all the manifestations. Holmes' thought that hypotonia might be such a fundamental disorder, but the mechanism by which hypotonia would explain other disorders has never been widely accepted, and recent evidence ${ }^{2}$ has demonstated that responses to stretch in many cerebellar patients is increased rather than decreased. Another candidate is dysmetria, movements that are abnormal by virtue of being the wrong amplitude, wrong direction, or wrong force. Dysmetria could be the principal explanation of other signs, such as dysdiadochokinesia and dyssynergia. Dysmetria is certainly a prominent abnormality of the cerebellar patient and one that causes significant functional disability. The purpose of this review is to consider what we know about dysmetria and to see how its pathophysiology might illuminate cerebellar physiology. Throughout, unless otherwise specified, the patients studied had diffuse cerebellar pathology and were chronically affected.

\section{Monophasic Movements at a Single Joint}

Rapid voluntary movements, such as quick monophasic movements at a single joint, provide a good approach to the study of movement, since they are accomplished in normal persons in a stereotypic fashion and with predictable timing. ${ }^{3-5}$ The pattern, sometimes called the "triphasic pattern," is characterized on the electromyogram (EMG) by an initial burst of muscle activity in the agonist of the movement that lasts 50 to $100 \mathrm{msec}$ (Figure 1). The agonist then falls silent and the antagonist muscle produces a burst that also lasts for 50 to $100 \mathrm{msec}$. Then the

From the Human Motor Control Section, Medical Neurology Branch, National Institute of Neurological Disorders and Stroke, National Institutes of Health, Bethesda, Maryland

Reprint requests to: Mark Hallett, M.D., Clinical Director, NINDS, National Institutes of Health, Building 10, Room 5N226, Bethesda, Maryland, USA 20892 
agonist again resumes activity, often in the form of a burst. For angular movements of less than 30 or 40 degrees, the timing of the movement changes only little; larger movements are accomplished with higher velocities. For movements of larger amplitude, the duration of the movement may increase and the EMG bursts are prolonged. ${ }^{6.7}$ Slow movements are characterized by long EMG bursts that parallel the duration of the movement. ${ }^{3}$

In an early study, ${ }^{8}$ we found that attempted rapid movements in patients with cerebellar deficits showed the triphasic pattern of EMG activity, but the duration of the first agonist burst was prolonged (Figure 1). We did not analyze the relationship between the EMG features and the kinematics of the movements, but speculated that this prolonged burst might predispose the patients to hypermetric movements, the most common type of dysmetria. The prolonged activity in the agonist muscle would move the limb for a longer time than was expected. However, there was a potential problem with this view. Because patients with cerebellar deficits often make movements that are hypermetric and slower than normal, and large-amplitude movements and slow movements are characterized by prolongation of the first agonist EMG burst, the patients may well have been making the wrong movement rather than a disordered movement. Therefore, we had to reconsider the question of whether these patients actually produce long first agonist bursts. ${ }^{9}$

We studied patients with cerebellar deficits in the arm, but no other neurologic signs. Patients and normal subjects were asked to make flexion movements of their elbows as rapidly as possible. They sat in a chair facing an oscilloscope screen with their arms strapped into a lightweight splint composed of an upper arm and forearm portion connected at the level of the elbow joint with a low-friction hinge. The beam on the oscilloscope screen was made into a line, the height of which indicated the elbow angle. Subjects were asked to make movements of 10,20 , and 30 degrees by specifying the beginning and ending heights of the line. The command to initiate movement was a verbal request to make the movement itself as rapidly as possible. Subjects were told that accuracy was less important than speed. The EMG was recorded with pairs of surface electrodes placed longitudinally over the bellies of the biceps brachii and triceps brachii. Some of the normal subjects were also asked to make movements at each distance with variable slow speeds or movements of 60 degrees, both rapidly and more slowly.

Normal subjects making rapid movements of 10,20, and 30 degrees had first agonist bursts averaging between 80 and 100 msec. Almost all patients had first agonist bursts that were significantly longer than normal for at least one movement distance, and analysis of variance showed a significant difference in burst duration between the normal subjects and the patients ( $p<0.001$ ). It is necessary, however, to assess burst durations for movements matched for distance and velocity. To do this, we looked at the first agonist burst duration for individual movements as a simultaneous function of angular distance and peak velocity (Figure 2). The three-dimensional plot of this relationship is a surface. The surface is flat and parallel to the plane of origin for rapid movements of less than 30 degrees. The edge of the surface, which comes from the most rapid movements that can be done for each distance, runs diagonally across the plane, because longer movements can be accomplished at faster velocity. The surface moves upward with longer or slower movements. In almost all patients at least some of the first agonist burst durations exceeded the normal distribution.

In comparison with the movements of the normal subjects, the patients' movements showed increased variability of distance moved for each distance aimed and marked hypermetria. Their movements were also slower than those of normal subjects. With division of the movement time into the acceleration time (beginning of the movement until the peak of the velocity) and the deceleration time (peak of the velocity until zero velocity), the most dramatic abnormality of the patients was a prolonged acceleration time $(p<0.001)$. This abnormality was also clear when the acceleration time was plotted as a simultaneous function of angular distance and peak velocity (Figure 2).

Rapid movements of normal subjects yielded a symmetric velocity curve, the acceleration time being roughly equal to the deceleration time (Figure 1). When making slow movements, they used a variety of strategies, but most commonly showed a

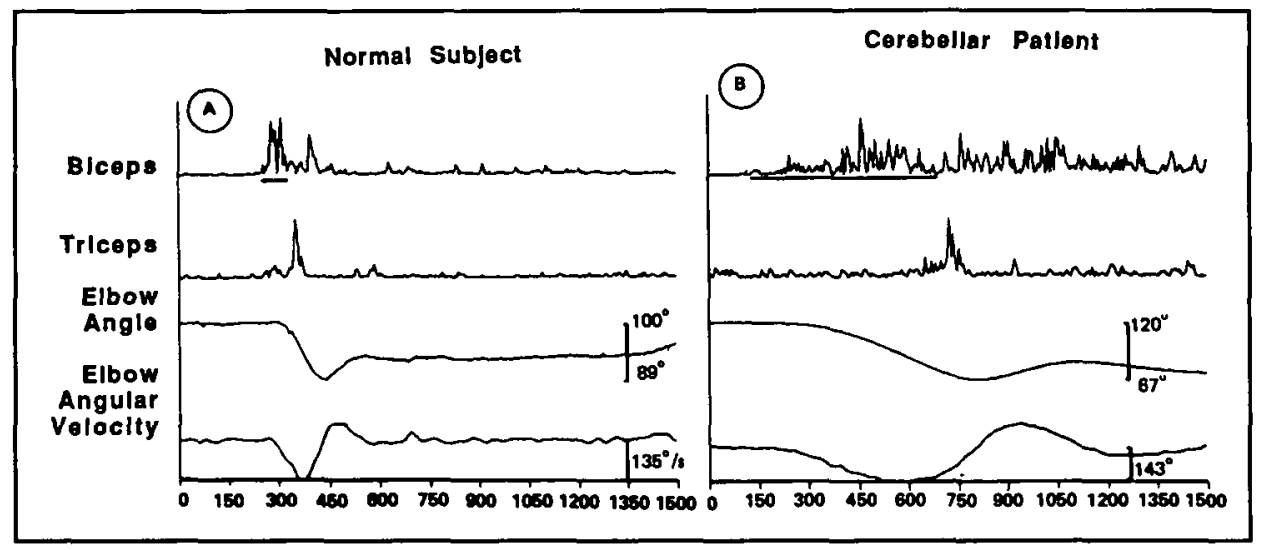

Figure $1-\boldsymbol{A}$ : Single elbow flexion movement of $11^{\circ}$ from a normal subject moving as fast as possible attempting to move $10^{\circ}$. B. Similarly recorded single elbow flexion movement of $53^{\circ}$ in a patient with cerebellar dysfunction attempting to move $30^{\circ}$. The four traces in each panel are, from top to bottom, rectified biceps EMG, rectified triceps EMG, angular position, and velocity. The line under the EMG of the biceps record indicates the measurement of the first agonist burst duration in that record. Modified from Hallett et al. ${ }^{9}$ 


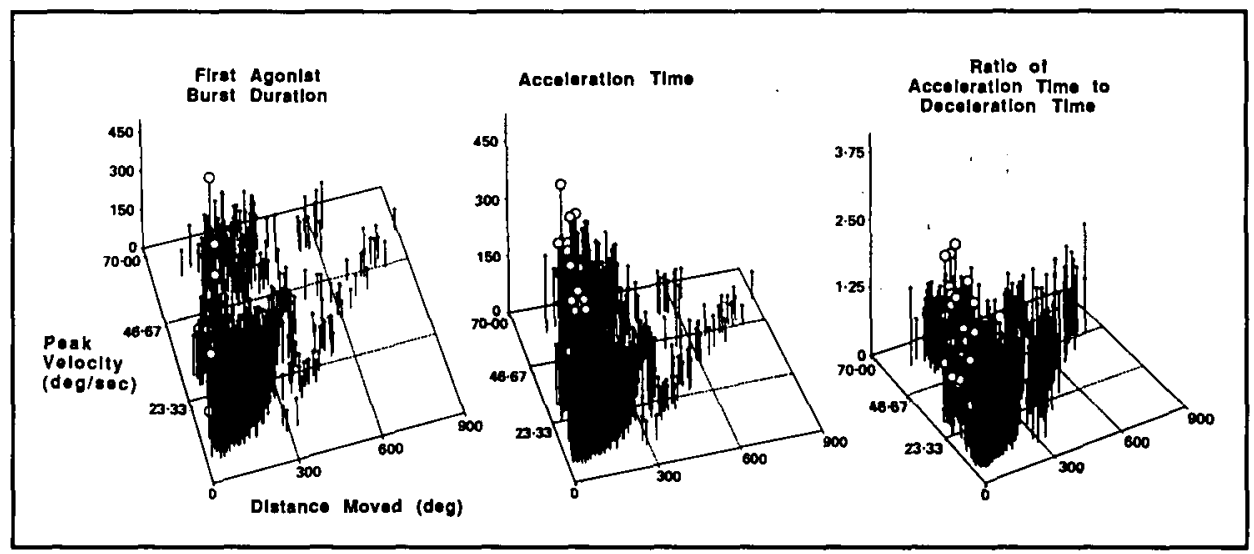

Figure 2-Left: Three-dimensional plot of duration of the first agonist burst as a function of distance moved and peak velocity. Data from normal subjects are plotted as small round points. Data from a cerebellar patient are plotted as large round points on the background of the normal data. Center: Three-dimensional plot of acceleration time as a function of distance moved and peak velocity. Data from normal subjects and the same patient as shown on the left are plotted as small and large round points, respectively. Right: Three-dimensional plot of the ratio of acceleration time to deceleration time as a function of distance moved and peak velocity. Data from normal subjects and the same patient as shown on the left are plotted as small and large round points, respectively. Modified from Hallett et al. ${ }^{9}$

longer deceleration time than acceleration time. The attempted rapid movements of the patients, however, often showed a longer acceleration time than deceleration time (Figure 1). To quantify these observations, we studied the ratio of acceleration time to deceleration time as a simultaneous function of angular distance and peak velocity (Figure 2). Again, most patients showed many abnormal movements.

Study of the relationship between the first agonist burst duration and kinematic parameters of the movement showed that the best correlate to the first agonist burst duration was the acceleration time (Figure 3).

Thus, the duration of the first agonist burst is prolonged in patients with cerebellar deficits, even when slowness of movement and hypermetria are considered. The correlation between the prolonged acceleration time and the prolonged EMG burst of the first agonist implies the logical view that the limb is accelerated for approximately the same time as the agonist EMG is active. The error of producing a long acceleration time would be a fundamental problem of simple movements for patients with cerebellar deficits. Hypermetria should be a common resultant movement error unless there is a compensatory reduction in the magnitude of the acceleration or a compensatory increase in the magnitude of the deceleration. It is important to realize that the abnormality identified in these studies can be appreciated even when controlled for distance and velocity of the movement. This means that the movement is the product of a deranged motor program and not an inappropriate normal program.

Similar findings have been made by Hore et al., ${ }^{10}$ who also studied rapid movements in patients with cerebellar disorders. Movements of 5, 30, and 60 degrees were studied at elbow, wrist, and finger with similar findings. Hypermetria was frequent and most dramatic for small aimed movements. The movement was asymmetric, with decreased magnitude of peak acceleration and increased magnitude of peak deceleration. The disorder of acceleration was associated with agonist EMG activ-

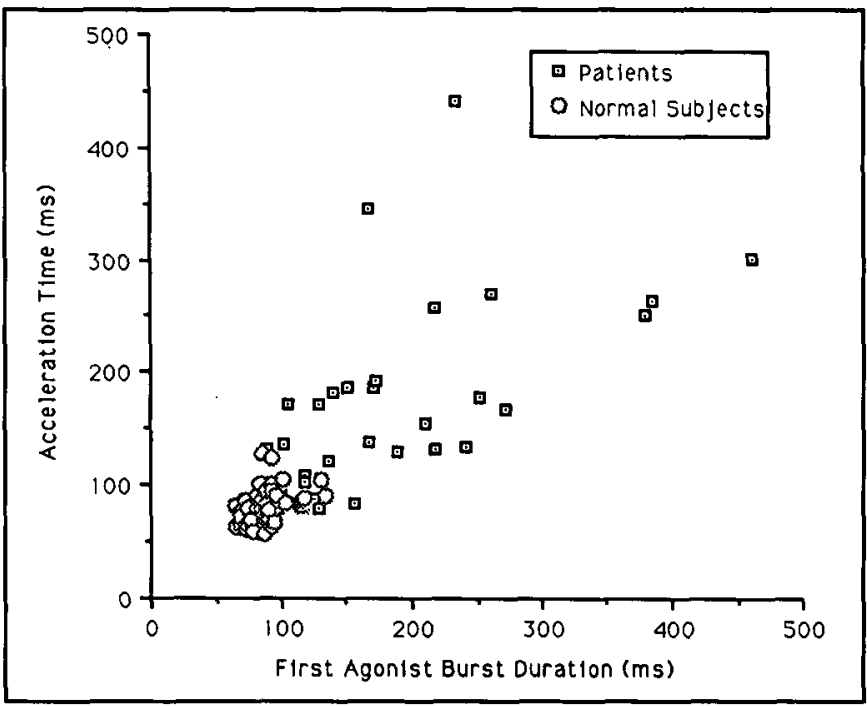

Figure 3 - Acceleration time as a function of agonist duration for patients and normal subjects using the mean values for distance aimed. Modified from Hallett et al. ${ }^{9}$

ity that was less abrupt in onset, smaller in magnitude, and more prolonged in duration. The disorder of deceleration was associated with delayed onset of phasic antagonistic EMG activity. Delay of the antagonist activity had previously been observed by Hallett et al. ${ }^{8}$

Brown et al. ${ }^{.1}$ have also carried out a similar kinematic study of elbow flexion movements of 10 to 70 degrees in patients with mild cerebellar dysfunction. Although they found abnormalities, they differed from those found later. ${ }^{9.10}$ The movement was asymmetric, with an acceleration phase shorter than the deceleration phase. The explanation for the difference appears to be that the movements were really different ones. The investigators urged their subjects to make accurate movements and omitted 
from the analysis any movements not within 3 degrees of the target. They claimed to have studied "fast movements," as well as "accurate movements," but the fast movements were studied after the accurate ones and were subject to the same accuracy requirements for analysis. Thus the movements were not as fast as those in the other studies. ${ }^{9.10}$ Additional support for this interpretation is that the asymmetry they observed was more pronounced when the movement time was increased. If this interpretation of the difference is correct, patients with cerebellar deficits may well have different types of derangements with different movements. Unfortunately, the EMG was not recorded in this study, so its characteristics in this situation are unknown.

Rapid voluntary movements have been studied in primate models of cerebellar dysfunction made by cooling the dentate nucleus (or other deep nuclei) with a probe. The animals show movements that resemble those of patients, with long first agonist bursts in simple movements ${ }^{12,13}$ or prolonged EMG bursts with alternating movements. ${ }^{14}$ Additionally, cooling the dentate nucleus produces prolongation of the phasic discharge of neurons in motor cortex that fire before the movement. ${ }^{15}$ An abnormally long acceleration time is found in the primate model. ${ }^{13,16}$ An abnormality of velocity in patients with cerebellar disease is in accord with studies of single-cell behavior in cerebellum recorded from performing primates. In several different circumstances, cellular activity has correlated with the velocity of movement as the best kinematic variable. ${ }^{17-19}$

\section{Termination of Movement and Simple Repetitive Move- ments}

Patients with cerebellar deficits also have abnormalities in termination of movement. This problem has been studied in a task where subjects were asked to make a rapid elbow flexion on the background of tonic elbow extension needed to hold a position against a background force. In this circumstance, the tonic triceps activity typically stops before the phasic biceps activity occurs (the "Hufschmidt phenomenon"). ${ }^{3}$ Patients with cerebellar dysfunction have a delay in terminating the triceps activity so that it overlaps the beginning of the biceps activity. ${ }^{8}$ This delay in stopping leads to overlap of the end of one movement with the beginning of the next. This kind of overlapping together with the abnormality of agonist burst duration should lead to clumsiness of successive movements, especially rapid, repetitive movements (dysdiadochokinesia).

\section{Reaction Time}

Abnormalities of reaction time would not immediately seem relevant to dysmetria, and they would not be for monophasic movements at a single joint. If coordinated movement at multiple joints is required, or if properly timed sequential movements are needed, however, accuracy in reaction time would be critical. Prolongation of reaction time for cerebellar patients was noted by Holmes' and verified by others. ${ }^{20}$ Prolongation of reaction time has also been found in animal models with cerebellar lesions. ${ }^{15,21,22}$

\section{Slow Movements}

Patients with cerebellar deficits show abnormalities in slow movements as well as rapid movements. Beppu et al. ${ }^{23}$ carefully analyzed slow, visually guided elbow tracking movements. They found delays in reaction time and in initiation of deceleration. At the onset of the movement, patients had difficulty in selecting an appropriate amplitude of peak velocity. During the movement, the trajectory was irregular rather than smooth. There was inaccuracy in corrective adjustment in reaching the final target point. The EMG activity, which should have been smooth and mainly in the agonist, was irregular and sometimes included excessive co-contraction of the antagonist. Beppu et al. ${ }^{24}$ later analyzed the irregularities in the mid-phase of the movement. During the tracking task, the visual signal reflecting either the arm position or the target was removed from view. This had no significant effect on normal subjects, but the patients with cerebellar deficits showed a reduction in the irregularity of the movement. The authors suggested that the perceived visual error between the target and the arm angle led to attempts at correction and thus the irregularity. When the error was no longer visible, the movement became smoother. Hence, some of the irregularity of movement may come from the patients' inability to deal appropriately with the visual feedback from the on-going movement.

\section{Multijoint Movements}

Although patients with cerebellar deficits have abnormalities in simple movements, they often appear to have even more trouble with complex movements involving multiple joints. Holmes ${ }^{1}$ noted the incoordination of compound movements that he called decomposition and asynergy. Such movements are now being studied in patients with cerebellar dysfunction.

Diener et al. ${ }^{25}$ have studied motor preparation in subjects who were asked to rise on their toes. This task requires a preparatory shift of the body forward with tibialis anterior activity and stabilization of the knee joint with quadriceps femoris activity prior to the execution of the task itself by triceps surae activation. The basic pattern of preparation and execution was preserved in 2 cerebellar patients, but changes in the relative timing of these two phases were sometimes seen. Similar studies were done by the same group, ${ }^{26}$ who examined the relationship of anticipatory postural activity with activity in the deltoid muscle with a bilateral arm elevation task. The temporal sequence was again disturbed.

Becker et al. ${ }^{27}$ have studied throwing a ball at a target in 3 patients with cerebellar dysfunction. Seated subjects, using an overhand throw, threw a tennis ball at a target 3 meters away. Elbow angle, wrist angle, and "hand opening angle" were measured using a two-camera Selspot II, and multiple muscles were monitored with EMG. Elbow-wrist coordination, hand direction, and the timing of hand opening were analyzed. The patients did not throw as accurately as normal subjects threw, and the same hand direction was not produced from trial to trial. In the assessment of elbow-wrist coordination, wrist flexion was properly timed after elbow extension, and wrist flexor EMG activity was properly delayed compared with triceps EMG activity. Normally, hand opening always occurred before peak distal forearm velocity, and this was maintained in cerebellar patients. The interval was shorter in all 3 patients than in any of the 3 normal subjects, but the difference was not statistically significant. The onset of EMG activity in the biceps and brachioradialis muscles was early relative to the triceps EMG activity and the kinematics of the movement. This may have contributed to the finding that the peak forearm velocity was late compared with the onset of elbow extension in all the patients. The authors concluded that, despite the inaccuracy of throwing, the measures of "coordination" were normal. This study appears promising, 
however, and the difficulty of finding abnormalities may relate to the small number of subjects studied so far and the relatively limited assessment.

Locomotion is a complex multijoint movement that is clearly abnormal in patients with cerebellar disturbances. We have recently begun biomechanical analyses of gait in patients with cerebellar disorders. ${ }^{28}$ Our studies utilized a video-based kinematic data acquisition system (VICON, Oxford Metrics, Inc.) for collection of unprocessed kinematic data from five video cameras. Reduction software (AMASS, Adtech) generated three-dimensional data, and the National Institutes of Health Automated Gait Evaluation Software (AGES) was used for data analysis. Retroreflective spherical targets were secured bilaterally to the skin surface at positions corresponding to the centroid of the temporalis muscle, greater tubercle of the humerus, lateral epicondyle of the humerus, posterior surface of the forearm midway between the styloid processes of the radius and ulna, greater trochanter of the femur, lateral femoral condyle, lateral malleolus, and fifth metatarsal-phalangeal joint. Patients and normal subjects were asked to walk normally across the laboratory. Our observations at this stage are only anecdotal.

Normal subjects walked in a straight line with constant step direction and stride length, but patients' steps lacked this constancy. No particular pattern could be discerned; both short and long steps occurred in no special order. Markedly abnormal steps were often associated with stumbling and even falls. The walking was not necessarily in a straight line and drifted to the right or left. Kinematic irregularities were apparent in measures of individual joints taken as a function of the gait cycle. In a gait cycle, time was normalized from $0 \%$ to $100 \%$, going from initiation of stance phase to end of swing phase. An example of the irregularity of joint movement is shown for the knee and the ankle in one subject (Figures 4 and 5). An important consistent abnormality in the kinematics was a delay in the flexion of the knee and the dorsiflexion of the ankle during early swing. Another abnormality of knee motion was the absence of the normal yield early in the stance phase. Another abnormality of ankle motion was the reduction in plantar flexion of the ankle in late swing.

More dramatic abnormalities in kinematics are illustrated with angle-angle diagrams, in which one joint angle is plotted by another. This illustrates the interlimb coordination, which was dramatically abnormal in the patients with cerebellar disease (Figure 6). Time is lost in these diagrams, but the time of each event in the gait cycle is ordinarily in the same place on the angle-angle diagram. Timing by this criterion was also deranged in the patients. Variability is apparent, but there are also consistent abnormalities. One consistent abnormality that can be detected in this type of display, not immediately apparent from the individual angle versus time graphs, is the relative delay of the knee flexion and ankle dorsiflexion. Swing begins at the lower right of each graph. With normal coordination, the curve moves up and to the left, but the patient's curve initially moves only to the left, signifying delay of knee motion relative to ankle motion. Thus, while both joint motions are delayed, they are not delayed the same amount, and the multijoint coordination is impaired.

We have also begun to look at multijoint coordination quantitatively in the upper extremity in a two-joint two-dimensional task. Cerebellar patients and normal subjects were asked to make straight left-to-right arm movements across a horizontal surface. Emphasis was placed on the straightness of the movement, and the subjects were coached to move at varying speeds to determine the effects of velocity on their performance. Patients were restrained at the torso and at the wrist so that hand movement was determined purely by rotations at the shoulder and elbow joints. Any disruption of the correct angular velocity ratios between the two joints would result in a deviation from linearity. The analysis was further simplified by paying attention primarily to the first $100 \mathrm{msec}$ of movement, the launch period. Within this period, conscious correction of movement errors is minimal if not absent.

The subjects sat facing a chest-high, horizontal digitizing tablet (Super L II, GTCO Corporation, Columbia, MD). Their torsos were restrained to a high-backed chair, and they wore wrist splints. Subjects used their dominant hand to hold an electronic stylus as if it were a pencil. The movement was conducted in darkness and cued by starting and target-direction light-emitting diodes (LED). However, patients were exhorted to pass beyond the target-direction LED without attempting to brake. This was done to minimize any possible interference of the launch command by an anticipated braking command. Kinematic information was derived from sampling of the position of the stylus. In the reference frame used, the positive

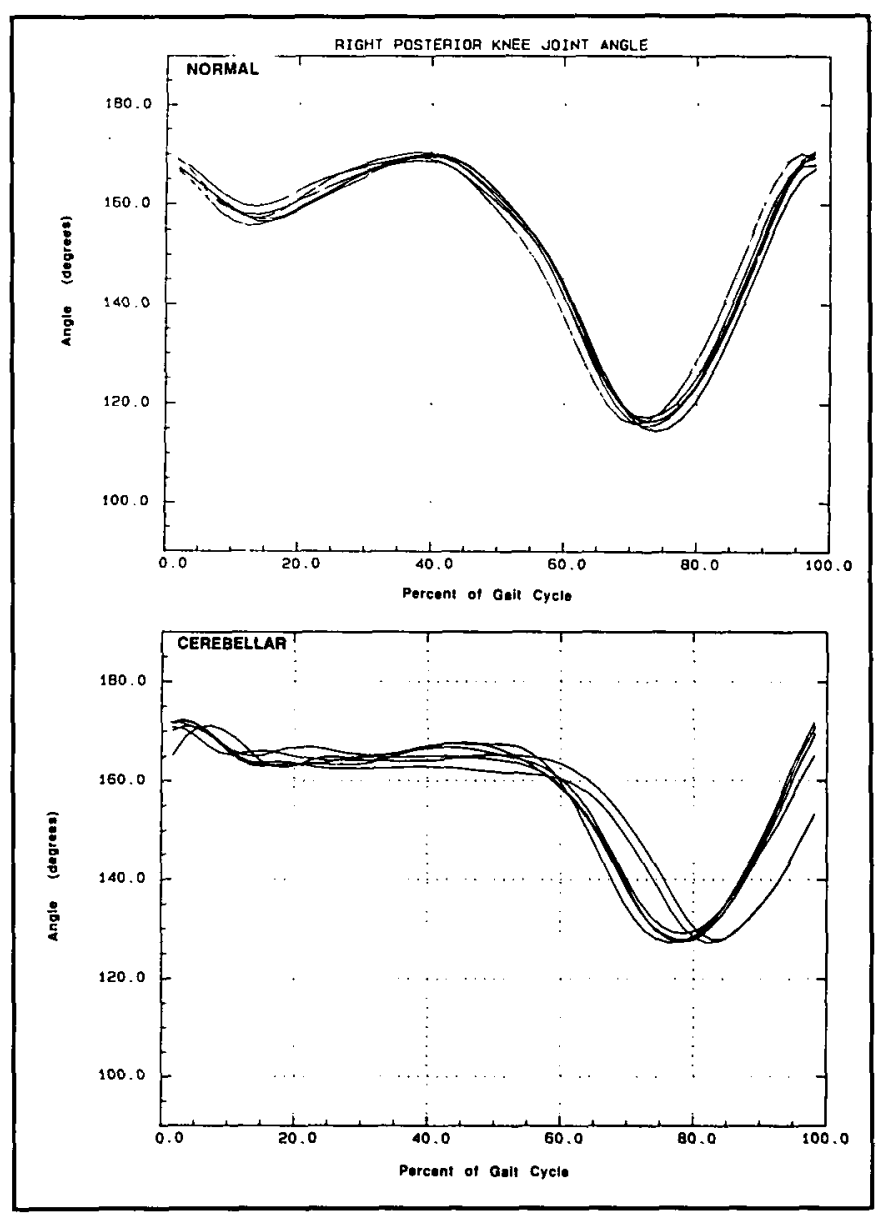

Figure 4-Movement of the knee in a normal subject (top) and a patient with cerebellar degeneration (bottom). Multiple trials are superimposed normalized to the gait cycle. 

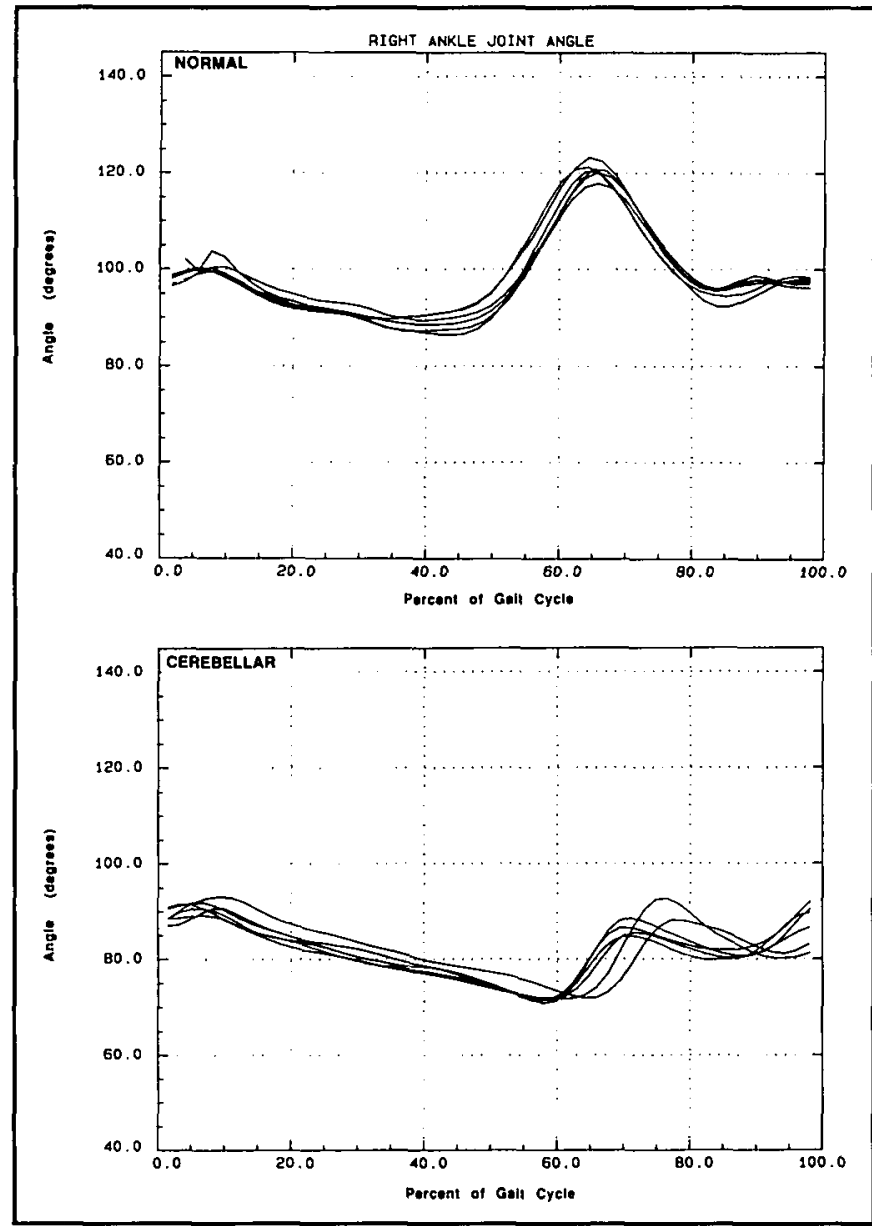

Figure 5 - Movement of the ankle in a normal subject (top) and a patient with cerebellar degeneration (bottom). Both the subject and the patient are the same as in Figure 4. Multiple trials are superimposed normalized to the gait cycle.

$\mathrm{X}$-axis extended to their right, and the positive $\mathrm{Y}$-axis extended forward away from their bodies. The shoulder angle was defined as the angle between the upper arm and the $\mathrm{X}$-axis. The value was positive for flexion. The elbow angle was defined relative to the axis of the upper arm; full extension at the elbow corresponded to zero degrees and the flexion direction was positive.

Representative trajectories from a normal subject and a moderately affected cerebellar patient are shown in Figure 7. At low speeds, normal subjects were able to produce fairly straight movements. At higher speeds, they exhibited a fairly characteristic disturbance. During the first $100 \mathrm{msec}$, their trajectories initially deviated toward their bodies, yielding a concavity. At some point after $100 \mathrm{msec}$, the concavity was followed by a mild convexity. The trajectory thereby assumed a sigmoidal shape and did not lose much overall directional accuracy. Cerebellar patients, however, tended to have more significant curvature within the first $100 \mathrm{msec}$ at all speeds. At the lowest speeds, they tended to show more variability in the pattern of curvature with a mixture of some mildly convex, straight, and mildly concave trajectories. They showed no significant abnormality at slow speeds. At any greater speed, and at lower speeds than in normal subjects, the concave pattern predominated. Unlike in normal subjects, however, the initial direction was not

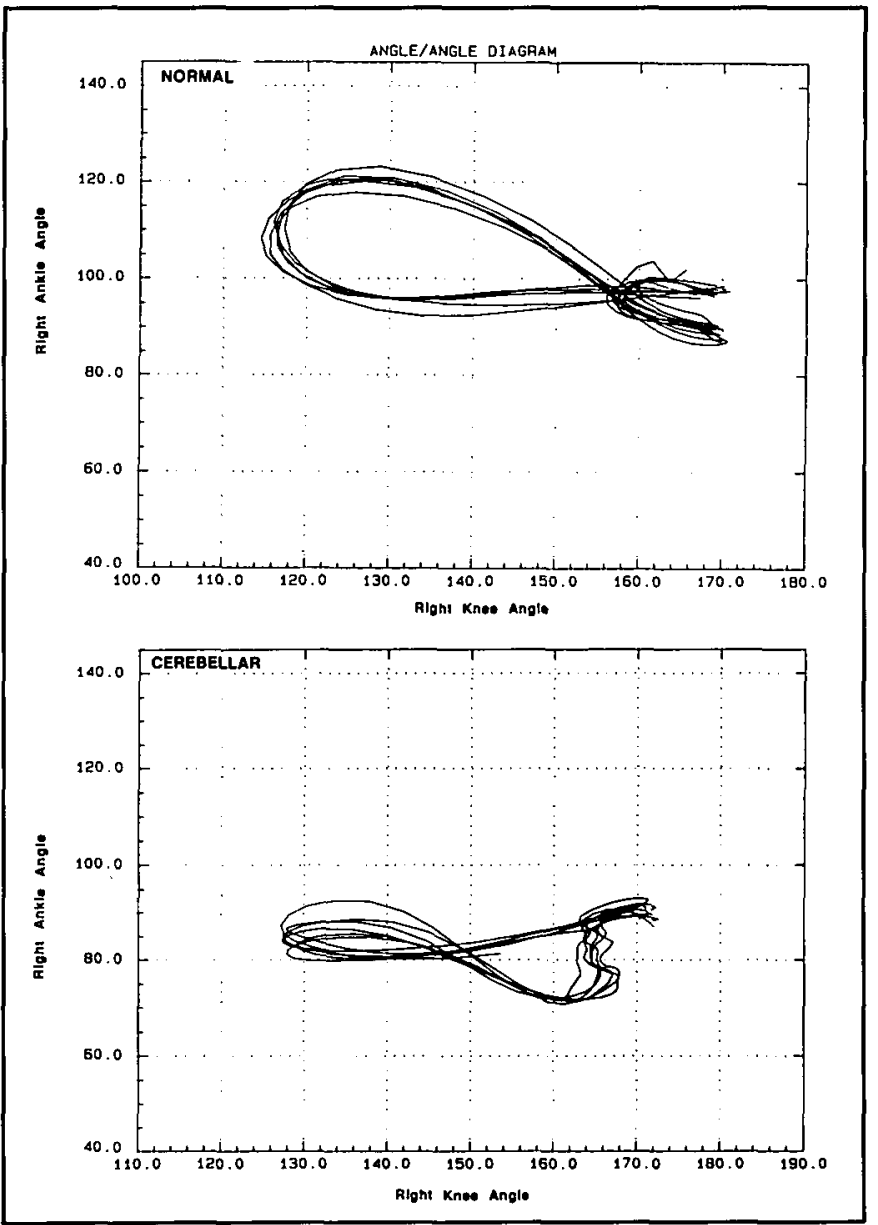

Figure 6-Movement of the knee plotted as a function of movement of the ankle (angle-angle diagram) in a normal subject (top) and a patient with cerebellar degeneration (bottom). Both the subject and the patient are the same as in Figure 4. Multiple trials are superimposed. For both the normal subject and the patient, the beginning and the end of the gait cycle is in the upper right part of the diagram. From the beginning, the traces first move to the lower right, then to the upper left, and finally return to the origin.

as strongly deviated toward the body, and in some cases may have been directly toward the target. Such an abnormality can be noted in Figure 7. Subsequent to the $100-\mathrm{msec}$ period, the trajectory of cerebellar patients typically became markedly convex with direction away from the target.

The source of the trajectory curvature can be appreciated from an examination of the joint rotation time courses shown in Figure 8. During the launch period, normal subjects exhibited relatively greater shoulder extension than elbow extension at all speeds. This tended to pull the hand more strongly in the $\mathrm{X}$ direction. At fast speeds, there even may have been slight elbow flexion, which further accentuated the $X$ component. As elbow extension began, more $\mathrm{Y}$ direction component was added, resulting in concavity. Eventually, elbow rotational velocity predominated. In the patients, the shoulder extension occurred more gradually and elbow extension predominated more quickly than in normal subjects, making the $X$ component of the launch less prominent. As the overall speeds of hand movements in these examples are close, but not identical, in the patients and 
normal subjects, care must be taken to regard the relative rates of action of the two joints and not their absolute time courses. Figure 8 shows abnormality of the fast movement, but not of the slow one. Figure 9 illustrates the joint coordination directly. Although elbow extension was excessive for both the slow and the fast movements of the cerebellar patient, it was present during the launch phase only for the fast movement.

These studies showed a velocity-dependent abnormality in multijoint coordination. The principal feature was excessive rotation of the elbow with respect to the shoulder. This was seen in normal subjects as they moved very rapidly, but possibly not until after the launch period. This was also seen in cerebellar patients with slow movements, but was very consistent with fast movements and typically characterized the first $100-\mathrm{msec}$ launch period. At this time, the abnormality must be considered a relative one. It is also possible that the elbow extension is inherently excessive or that the shoulder extension is inherently deficient.

\section{Sensory Ataxia}

A point of confusion in thinking about the cerebellum and dysmetria is that patients with severe sensory loss may also show ataxia said to be clinically similar to that shown by patients with cerebellar lesions. ${ }^{29.30}$ The characteristics of the two movement disorders, however, show them to be different. Many practiced movements in controlled situations can be done quite accurately in patients with sensory loss. ${ }^{31.32}$ Patients with sensory loss show instability of postural maintenance that may mimic athetosis. ${ }^{32}$ Vision is extraordinarily helpful to patients with sensory loss, ${ }^{31,32}$ but may hinder patients with cerebellar dysfunction. ${ }^{24}$ Movements encompassing somatosensory reflexes are diminished in patients with sensory loss, ${ }^{32}$ but may be excessive in patients with cerebellar dysfunction. ${ }^{2}$

Kinematic and EMG analysis of the sort described here for patients with cerebellar dysfunction has not been done for patients with sensory loss. A preliminary report by Sainburg et al. ${ }^{33}$ on multijoint movement in a patient with sensory loss has shown lack of synchronization of elbow and shoulder joint motions. Careful comparison between the abnormalities in cerebellar dysfunction and sensory loss will probably be most helpful in defining the importance of feedback to cerebellar function.

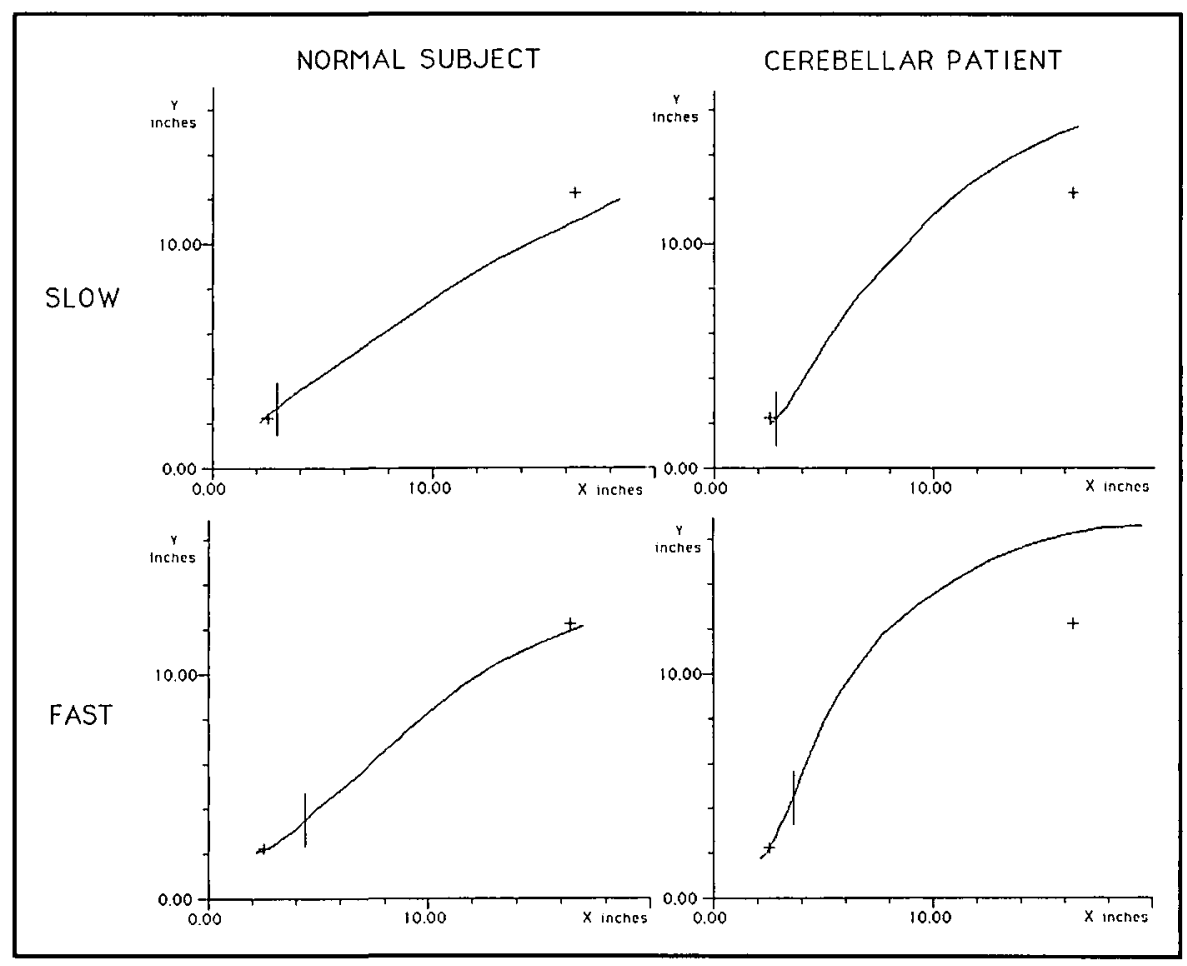

Figure 7 - Trajectories of the right hands of a normal subject and a cerebellar patient attempting to produce linear paths across the horizontal $(X-Y)$ plane. The movements travel from left to right and away from the body; the starting and target-direction light-emitting diode positions are indicated by + . Slow and fast movements having respective peak velocities of 8 to 18 inches/ second and 85 to 97 inches/second are compared. The initial $400 \mathrm{msec}$ of each trajectory is shown for the slow movements. All acquired data points are shown for fast movements that left the recording surface in under $200 \mathrm{msec}$. The position at $100 \mathrm{msec}$ is indicated by a short vertical line. The normal subject shows a gentle, but consistent sigmoidicity, first concave then convex relative to the body. The cerebellar patient demonstrates significant sigmoidicity at both speeds but with a more pronounced convex portion. At high speeds, the effect is exaggerated and the movement becomes almost a simple arc, but the initial concavity persists. The net effect is one of increasing angular discrepancy from the target direction with increasing velocity. 


\section{Deficiency in the Rate of Change of Force}

Dysmetria is an important abnormality in a wide range of motor behaviors of patients with cerebellar dysfunction. It is not unreasonable, therefore, to consider it as a fundamental disturbance of cerebellar disruption. What is the general explanation for dysmetria? One idea is that there is a deficiency of force generation. The deficiency, if there is one, is not in the ability of the patient to generate peak force, although Holmes ${ }^{l}$ did note this sign (asthenia) in many of his patients. The abnormality would be an inability to generate force with appropriate speed, that is, the rate of change of force. In the monophasic movements at a single joint, EMG activity has a very slow onset and acceleration is reduced. If the increase in force is slow, then the force must be generated for a longer period of time to produce the desired movement. This might lead to a compensatory longer burst of initial EMG activity, a long acceleration time, and a slow movement. Disruption of subsequent timing would also result. This could explain multijoint incoordination if there is a greater slowness of force at one joint than at another. For

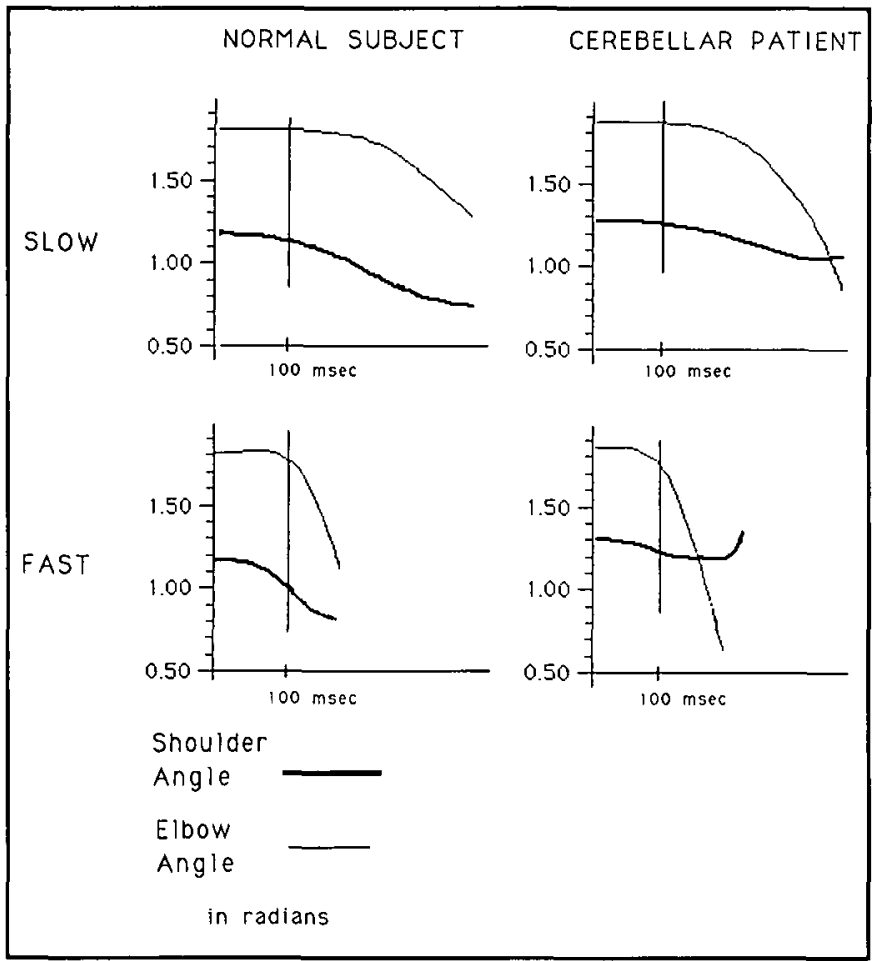

Figure 8-Time courses of shoulder and elbow angles are compared with attention focused on the initial 100-msec interval. These data are from the same movements illustrated in Figure 7. Initial values are determined by the subjects' limb segment lengths and positioning relative to the starting point. Because the overall hand speeds were close, but not identical, in these examples, attention is called to the relative behavior of the shoulder and elbow. rather than to their absolute time courses. Within the first 100 msec, all movements produced consist principally of extension at both joints. In detail, however, the normal subject show's a very small amount of initial elbow flexion at high speeds and in general, at all speeds. shows considerably less net elbow extension than shoulder extension. By contrast, the cerebellar patient, at low' speed, shows only slightly less relative elbow extension, and at high speed, elbow extension predominates slightly. The relative preponderance of elbow activity in the patient corresponds to the more pronounced convex portion of the planar trajectory. example, in our studies of two-joint straight movements, the relative deficiency at the shoulder could be due to a greater deficit in force generation there compared with the elbow. Another abnormality that may support this notion is the finding of Mai et al. ${ }^{34}$ that patients with cerebellar lesions have difficulties in maintaining constant force. This skill should depend on the ability to alter force both continuously and quickly to correct errors. Limitation of force generation would explain why patients have much more difficulty with fast movements than with slow ones, because demands for force generation are much greater with the fast movements. If this is the fundamental explanation for dysmetria, then it suggests a normal role of the cerebellum in setting the rate of change of force. There is recent evidence ${ }^{35}$ that minimization of torque change is the best descriptor of optimal trajectories, so this would be consistent with the cerebellum's central role in coordination. Further work in this area might include studies of motor unit recruitment in cerebellar patients.

\section{Deficiency in Timing}

Another possible explanation for dysmetria is that there is an abnormality in the timing of motor behavior. This is a concept that has been widely suspected for many years. The analogy has often been made between the cerebellum and a clock. ${ }^{36-38}$ Such an abnormality could explain many of the phenomena of dysmetria. Timing of movement onset is deranged, both in reaction time and velocity (or acceleration), which is the derivative of position with respect to time. Timing of movement termination is abnormal. Failure to terminate the first agonist EMG burst properly could lead to excessive duration; decreased amplitude and rate of rise of EMG could then be compensatory to avoid excessive force. Multijoint movements require more exquisite

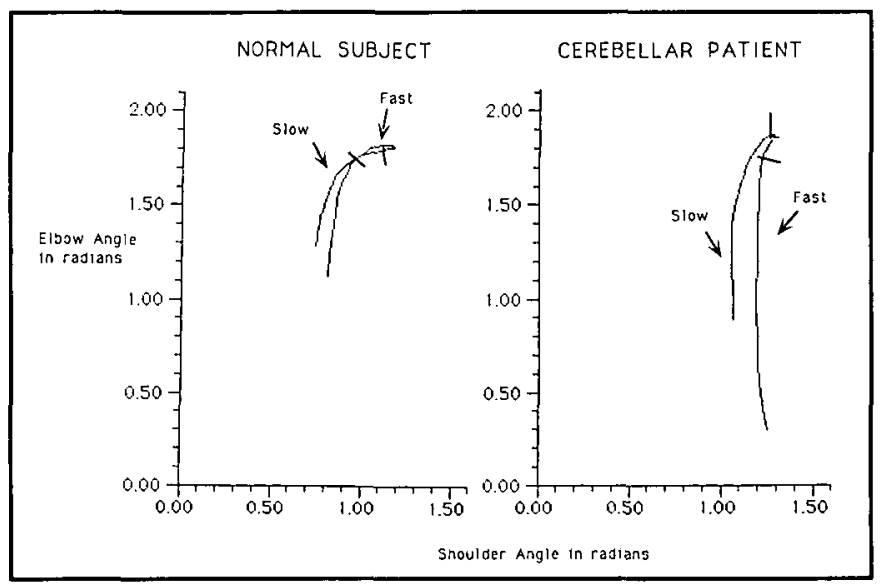

Figure 9 - The coordination between the elbow and shoulder joints is summarized by plotting the trajectory in joint space. These data are from the same movements illustrated in Figure 7. The paths travel from larger to smaller joint angles beginning from the same starting points. The 100-msec point is indicated by a short perpendicular line. The normal subject show's a pattern of significant shoulder extension prior to elbow extension. Because at low speed the normal subject produces a nearly ideal straight path toward the target, the low-speed curve represents nearly the ideal joint coordination pattern. At high speeds, there is disproportionate elbow extension preceded by a slight elbow flexion. The cerebellar patient also show's sequential extension at the shoulder and then the elbow. However, elbow extension comes to predominate more dramatically. and elbow flexion is not typically seen. 
timing than monophasic, single-joint movements, as the relative timing of the different joints to each other plays an important role. Hence, an abnormality in timing would make multijoint movements appear more impaired than single-joint movements. A fundamental abnormality of timing might explain more than an abnormality of force generation. Moreover, the abnormality of force generation might be conceived as a secondary consequence, since force change is a time derivative of force, or as a compensation to minimize the effects of timing disturbance. Indeed, many patients with cerebellar deficits prefer to move slowly even when they can move more rapidly. Further work in this area might seek to demonstrate more derangement with more exacting demands of timing.

There is other evidence for a central role of the cerebellum in timing of motor behavior. ${ }^{39}$ Patients with cerebellar dysfunction and normal subjects were asked to tap a constant rhythm. With a theoretical model of this behavior ${ }^{40}$ errors in time-interval production could be analyzed for disorders of a central pacemaker and for disorders of timing of implementation of each tap. Cerebellar patients were abnormal on both components. In a subsequent study, ${ }^{41}$ cerebellar patients with focal lesions were investigated. Patients with lateral hemispheric lesions apparently had specific dysfunction of the central pacemaker, whereas patients with medial lesions had specific dysfunction of the implementation component.

Further support of a role for the cerebellum as a clock comes from studies on the perception of time intervals. Cerebellar patients have a deficit in assessing the relative duration of acoustic and visual time intervals. ${ }^{39.42} \mathrm{~A}$ direct implication of this finding for motor behavior is that feedback from an ongoing movement may be misinterpreted, and corrective movements would be subsequently incorrect. This may explain findings such as those of Beppu et al., ${ }^{23.24}$ discussed earlier, where feedback was not handled well and tracking became smoother when feedback was eliminated.

Studies of dysmetria can be used as support for the concept of the normal role of the cerebellum in timing activities of the brain. Timing is so critical for many operations that such a role may well be its most important function.

\section{ACKNOWLEDGMENTS}

We are grateful to S.L. Thomas and S.J. Stanhope for helping with the gait analysis and to B.J. Hessie for skillful editing.

\section{REFERENCES}

1. Holmes G. The cerebellum of man. Brain 1939; 62: 1-30.

2. Friedemann $\mathrm{HH}$, Noth J, Diener $\mathrm{HC}$, et al. Long latency EMG responses in hand and leg muscles: cerebellar disorders. J Neurol Neurosurg Psychiatry 1987; 50: 7 1-77.

3. Hallett M, Shahani BT, Young RR. EMG analysis of stereotyped voluntary movements in man. J Neurol Neurosurg Psychiatry 1975; 38: 1154-1162.

4. Freund H-J, Büdigen HJ. The relationship between speed and amplitude of the fastest voluntary contractions of human arm muscles. Exp Brain Res 1978; 1: 1-12.

5. Hallett M, Marsden CD. Ballistic flexion movements of the human thumb. J Physiol (Lond) 1979; 294: 33-50.

6. Berardelli A, Rothwell JC, Day BL, et al. Duration of the first agonist EMG burst in ballistic arm movements. Brain Res 1984; 304: 183-187.

7. Brown SH, Cooke JD. Initial agonist burst duration depends on movement amplitude. Exp Brain Res 1984; 55: 523-527.
8. Hallett $M$, Shahani BT, Young RR. EMG analysis of patients with cerebellar deficits. J Neurol Neurosurg Psychiatry 1975; 38: 1163-1169.

9. Hallett M, Berardelli A, Matheson J, et al. Physiological analysis of simple rapid movements in patients with cerebellar deficits. $J$ Neurol Neurosurg Psychiatry 1991; 53: 124-133.

10. Hore J, Wild B, Diener HC. Cerebellar dysmetria at the elbow, wrist and fingers. J Neurophysiol 1991; 65: 563-571.

11. Brown $\mathrm{SH}$, Hefter $\mathrm{H}$, Mertens $\mathrm{M}$, et al. Disturbances in human arm movement trajectory due to mild cerebellar dysfunction. J Neurol Neurosurg Psychiatry 1990; 53: 306-313.

12. Meyer-Lohmann J, Conrad B, Matsunami K, et al. Effects of dentate cooling on precentral unit activity following torque pulse injections into elbow movements. Brain Res 1975; 94: 237-251.

13. Flament D, Hore J. Movement and electromyographic disorders associated with cerebellar dysmetria. J Neurophysiol 1986; 55: 1221-1233.

14. Conrad B, Brooks VB. Effects of dentate cooling on rapid alternating arm movements. J Neurophysiol 1974; 37: 792-804.

15. Meyer-Lohmann J, Hore J, Brooks VB. Cerebellar participation in generation of prompt arm movements. J Neurophysiol 1977; 40: 1038-1050.

16. Brooks VB, Kozlovskaya IB, Atkin A, et al. Effects of cooling dentate nucleus on tracking-task performance in monkeys. $J$ Neurophysiol 1973; 36: 974-995.

17. Robertson LT, Grimm RJ. Responses of primate dentate neurons to different trajectories of the limb. Exp Brain Res 1975; 23: 447462.

18. Thach WT. Correlation of neural discharge with pattern and force of muscular activity, joint position, and direction of intended next movement in motor cortex and cerebellum. J Neurophysiol 1978; 41: 654-676.

19. Burton JE, Onoda N. Interpositus neuron discharge in relation to a voluntary movement. Brain Res 1977; 121: 167-172.

20. Marsden CD, Merton PA, Morton HB, et al. Disorders of movement in cerebellar disease in man. In: Rose FC, ed. Physiological Aspects of Clinical Neurology. Oxford: Blackwell, 1977: 179. 199.

21. Lamarre Y, Jacks B. Involvement of the cerebellum in the initiation of fast ballistic movement in the monkey. Electroencephalogr Clin Neurophysiol 1978: 34 (Suppl): 441-447.

22. Miller AD, Brooks VB. Parallel pathways for movement initiation in monkeys. Exp Brain Res 1982; 45: 328-332.

23. Beppu H, Suda M, Tanaka R. Analysis of cerebellar motor disorders by visually guided elbow tracking movement. Brain 1984; 107: 787-809.

24. Beppu H, Nagaoka M, Tanaka R. Analysis of cerebellar motor disorders by visually guided elbow tracking movement. 2. Contribution of the visual cues on slow ramp pursuit. Brain 1987; 110: 1-18.

25. Diener HC, Dichgans J, Guschlbauer B, et al. Associated postural adjustments with body movement in normal subjects and patients with parkinsonism and cerebellar disease. Rev Neurol (Paris) 1990; 146: 555-563.

26. Diener HC, Dichgans J, Guschlbauer B, et al. Disturbances of motor preparation in basal ganglia and cerebellar disorders. Prog Brain Res 1989; 80: 481-488; discussion 479-480.

27. Becker WJ, Kunesch E, Freund H-J. Coordination of a multijoint movement in normal humans and in patients with cerebellar dysfunction. Can J Neurol Sci 1990; 17: 264-274.

28. Hallett M, Stanhope SJ, Thomas SL, et al. Pathophysiology of posture and gait in cerebellar ataxia. In: Shimamura $M$, Grillner $S$, Edgerton VR, eds. Neurobiological Basis of Human Locomotion. Tokyo: Japan Scientific Societies Press, 1991: 275283.

29. Dalakas MC. Chronic idiopathic ataxic neuropathy. Ann Neurol 1986; 19: 545-554.

30. Taly AB, Prasad A, Vasanth A, et al. Acute ataxic neuropathy: a clinical, electrophysiological and morphological study. Acta Neurol Scand 1991; 84: 398-402.

31. Rothwell JC, Traub MM, Day BL, et al. Manual motor performance in a deafferented man. Brain 1982; 105: 515-542. 
32. Sanes JN, Mauritz KH, Evarts EV, et al. Motor deficits in patients with large-fiber sensory neuropathy. Proc Natl Acad Sci USA 1984; 81: 979-982.

33. Sainburg RL, Poizner H, Ghez C. Loss of proprioception produces deficits in interjoint coordination. Soc Neurosci Abstr 1991; 17(part 2): 1386.

34. Mai N, Bolsinger $P$, Avarello $M$, et al. Control of isometric finger force in patients with cerebellar disease. Brain 1988; 111: 973998.

35. Uno Y, Kawato M, Suzuki R. Formation and control of optimal trajectory in human multijoint arm movement. Minimum torquechange model. Biol Cybern 1989; 61: 89-101.

36. Braitenberg V. Is the cerebellar cortex a biological clock in the millisecond range? Prog Brain Res 1967; 25: 334-346.

37. Kornhuber HH. Motor functions of cerebellum and basal ganglia: the cerebello-cortical saccadic (ballistic) clock, the cerebellonu- clear hold regulator, and the basal ganglia ramp (voluntary speed smooth movement) generator. Kybernetik 1971; 8: 157-162.

38. Eccles J. Cerebellar function in the control of movement. In: Rose FC, ed. Physiological Aspects of Clinical Neurology. Oxford: Blackwell Scientific, 1977: 157-178.

39. Ivry R, Keele SW. Timing functions of the cerebellum. J Cogn Neurosci 1989; 1: 136-152.

40. Wing AM, Kristofferson AB. Response delays and the timing of discrete motor responses. Perception Psychophysics 1973; 14: 5 12.

41. Ivry RB, Keele SW, Diener HC. Differential contributions of the lateral and medial cerebellum to timing and to movement execution. Exp Brain Res 1988; 73: 167-180.

42. Keele SW, Ivry R. Does the cerebellum provide a common computation for diverse tasks? A timing hypothesis. Ann NY Acad Sci 1990; 608: 179-207; discussion 207-211. 